\title{
CINÉTICA DE CRESCIMENTO FÚNGICO EM CULTIVO SÓLIDO POR TEOR DE N- ACETILGLICOSAMINA E ATIVIDADE ENZIMÁTICA
}

\author{
A.C.S.GOMES ${ }^{1 *}$, F.P.CASCIATORI ${ }^{2}$, E. GOMES ${ }^{3}$, J.C.THOMÉO ${ }^{1}$ \\ ${ }^{1}$ Universidade Estadual Paulista "Júlio de Mesquita Filho", Departamento de Engenharia e \\ Ciência dos Alimentos \\ ${ }^{2}$ Universidade Estadual Paulista "Júlio de Mesquita Filho", Departamento de Engenharia e Ciência \\ dos Alimentos \\ ${ }^{3}$ Universidade Estadual Paulista "Júlio de Mesquita Filho", Departamento de Biologia \\ *e-mail: carolsantos218@hotmail.com
}

\begin{abstract}
RESUMO
O objetivo do presente trabalho foi avaliar a cinética de crescimento do fungo termófilo Myceliophthora thermophila M.7.7 em cultivo sólido. A aplicação imediata desta cinética é empregá-la a modelos de previsão de transferência de calor e massa em biorreatores; em horizonte mais amplo, a cinética poderá ser relacionada à secreção de metabólitos pelo microrganismo. $\mathrm{O}$ meio de cultivo foi composto por bagaço de cana de açúcar e farelo de trigo (proporção 7:3) e os métodos analíticos se basearam nas quantificações de $\mathrm{N}$-acetilglicosamina e atividade de CMCase, $\alpha$ amilases, xilanase e proteases. A técnica de análise do teor de $\mathrm{N}$-acetilglicosamina mostrou ser um bom indicativo de crescimento microbiano, com bom ajuste ao modelo logístico, resultando em um coeficiente $\mathrm{R}^{2}$ igual a 0,98 , tendo permitido estimar os parâmetros cinéticos de crescimento, onde o Xmáx foi de 1,889 mg de glicosamina/g de fermentado. A análise da cinética de secreção das enzimas estudadas foi um bom indicador das atividades metabólicas do fungo, mas não de seu crescimento.
\end{abstract}

\section{INTRODUÇÃO}

Os cultivos em estado sólido (CES) se caracterizam por utilizarem substratos insolúveis, em geral resíduos agroindustriais, e reduzida quantidade de água. A maioria dos processos em meio sólido são conduzidos por fungos filamentosos, cujo crescimento acontece pela extensão das pontas das hifas sobre a superfície sólida (LONSANE et al., 1999). A aplicação comercial do CES encontra algumas limitações no design e operação de biorreatores em larga escala, tais como remoção do calor gerado pelo metabolismo microbiano e heterogeneidade da mistura dos materiais sólidos. Este último aspecto dificulta o controle do crescimento celular, de temperatura e das concentrações de nutrientes e produtos, o que torna complexo o controle e automação do processo. Para superar tais dificuldades, uma ferramenta útil para o desenvolvimento de fermentadores para CES é a simulação do processo para a compreensão dos fenômenos de transferência de calor e massa e previsões de consumo de nutrientes e secreção de metabólitos. Para a aplicação destes modelos de previsão requerse a cinética de crescimento da biomassa microbiana. Entretanto, a determinação direta de biomassa em CES é inviável devido à impossibilidade de separação completa entre biomassa microbiana e substrato, particularmente para processos envolvendo fungos, pois suas hifas aderem fortemente ao 
suporte sólido. A literatura reporta alguns métodos indiretos de quantificação da biomassa baseado nos constituintes celulares, como a quitina, ergoesterol, ácidos nucléicos e proteínas, assim como na determinação da atividade biológica, tais como ATP, atividade enzimática, taxa de respiração e consumo de nutrientes. Dentro desse contexto e diante da necessidade de se prover desenvolvedores e operadores de sistemas de cultivo em estado sólido com modelos cinéticos confiáveis, o presente trabalho visou estimar a cinética de crescimento do fungo termofílico Myceliophthora thermophila M.7.7 sendo aplicadas as técnicas indiretas de determinação de biomassa.

\section{MATERIAL E MÉTODOS}

\subsection{Microrganismo e condições de cultivo}

A linhagem do fungo termofílico Myceliophthora thermophila M.7.7 foi mantida em tubos de ensaio contendo meio de cultivo Agar-SabouraudDextrose (ASD) inclinado, submerso em óleo mineral, mantendo-se os tubos a $5^{\circ} \mathrm{C}$. O monitoramento da cinética do fungo foi estudado em meios de cultura com a seguinte composição: bagaço de cana e farelo de trigo na proporção 7:3 e a solução nutriente foi composta por: sulfato de amônio (1\%); MAP (3 g / L); cloreto de potássio (2 g / L); MgSO4.7H2O (0,5 g / L); $\mathrm{CaCl} 2(0.1 \mathrm{~g} / \mathrm{L})$ e Tween $80(0,1 \% \mathrm{v} / \mathrm{v})$, a $\mathrm{pH} 5$.

\subsection{Determinação de $\mathbf{N}$-acetilglicosamina}

$\mathrm{Na}$ quantificação do teor de glicosamina no material fermentado, foi empregado o método descrito por Aidoo, Hendry e Wood (1981) e Dalsenter (2005).

\subsection{Correlações entre a biomassa e o conteúdo de glicosamina em cultivo líquido}

A fim de obter uma correlação direta entre o micélio seco e a concentração de glicosamina, o fungo foi inoculado em cultivo submerso (Csb) e a partir de diferentes concentrações do peso seco foi determinado o teor de glicosamina. $\mathrm{O}$ meio de cultivo foi composto por: amido de mandioca $(10 \mathrm{~g} / \mathrm{L})$; extrato de levedura $(5 \mathrm{~g} / \mathrm{L})$; peptona $(2 \mathrm{~g} /$ $\mathrm{L}) ;\left(\mathrm{NH}_{4}\right)_{2} \mathrm{SO}_{4}(2 \mathrm{~g} / \mathrm{L}), \mathrm{K}_{2} \mathrm{HPO}_{4}(7 \mathrm{~g} / \mathrm{L}) \mathrm{e}$ $\mathrm{MgSO}_{4} \cdot 7 \mathrm{H}_{2} \mathrm{O}(0,1 \mathrm{~g} / \mathrm{L})$, a $\mathrm{pH} 5$.

A determinação do peso seco foi realizada por secagem do material obtido, após filtração à vácuo em papel de filtro previamente seco e pesado, em estufa a $100^{\circ} \mathrm{C}$ por 24 horas. O teor de glicosamina do micélio seco foi determinado conforme o item anterior. Os valores obtidos foram utilizados para determinar a relação entre massa seca do fungo e conteúdo de glicosamina, que foi utilizada, posteriormente, na determinação da massa celular obtida nos cultivos em estado sólido. Os resultados foram expressos em $\mathrm{mg}$ de glicosamina por $\mathrm{g}$ de biomassa seca nos cultivos líquidos e em mg de glicosamina por $\mathrm{g}$ de meio sólido, nos cultivos em meio sólido.

\subsection{Modelagem da cinética do crescimento microbiano em CES}

A cinética de crescimento dos microrganismos em CES tem sido comumente expressa como uma cinética de primeira ordem ou, preferencialmente, como uma equação logística, que é um modelo nãoestruturado empírico e baseado em observações experimentais, no qual é possível descrever as diferentes sensibilidades dos microrganismos em função da temperatura. A despeito da simplicidade matemática, a equação logística pode representar uma aproximação adequada da curva de crescimento completa em uma única equação, incluindo as fases lag, de crescimento exponencial e estacionária (FANAEI; VAZIRI, 2009), sendo representada por: 


$$
\frac{d X}{d t}=\mu X\left(1-\frac{X}{X_{m}}\right)
$$

onde $\mathrm{X}$ é a concentração de biomassa microbiana no substrato e $X_{m}$ seu valor máximo; e $\mu$ é a taxa de crescimento específico.

A condição inicial adotada para solução da equação é: para $\mathrm{t}=0, \mathrm{X}=\mathrm{X}_{0}$ (concentração de biomassa inoculada no início do processo). Assim, a forma integrada do modelo logístico foi apresentada por Dalsenter (2005) como segue:

$$
X=\frac{X_{m}}{1+\left(\frac{X_{m}}{X_{0}}-1\right) e^{-\mu t}}
$$

\subsection{Análises estatísticas}

Nas determinações analíticas, as amostras necessárias são muito reduzidas, de modo que precauções foram tomadas para que os resultados fossem estatisticamente conclusivos. Para avaliar esta possível influência, foram feitas cinco repetições independentes de cultivo sólido para cada análise das propriedades determinadas (Nacetilglicosamina e atividade enzimática).

Os cálculos foram realizados no software Origin versão 6.0 (Microcal Software Inc., USA). Análises de dispersão, tais como desvio padrão para a média, intervalo de confiança e coeficiente de variação foram calculados em planilha Excel (Microsoft 2008). Comparações entre médias foram feitas através do teste de Tukey empregando-se o software Minitab, versão 16.0. E comparação entre as variâncias foram feitas através do teste de Levene.

\subsection{Determinação da atividade enzimática}

Foram determinadas a atividades das enzimas CMCase, $\alpha$-amilases e proteases e xilanases ao longo do tempo de cultivo. As atividades de CMCase, xilanase e alfaamilase, foram determinadas utilizando o método proposto por Ghose (1987), que se baseia na determinação de açúcares redutores totais. A reação foi mantida a $70^{\circ} \mathrm{C}$ por 10 min., sendo, interrompida pela adição de 1,0 $\mathrm{mL}$ do reagente DNS (ácido 3-5dinitrosalicílico) para a quantificação dos açúcares redutores liberados.

A atividade de proteases foi determinada de acordo com Merheb et al. (2007), com modificações. A reação foi mantida a $35^{\circ} \mathrm{C}$ e ao final de 30 minutos a reação foi interrompida pela adição de $1 \mathrm{~mL}$ de TCA (ácido tricloroacético) 10\%.

\section{RESULTADOS E DISCUSSÃO}

\subsection{Correlação entre biomassa e teor de $\mathbf{N}$ - acetilglicosamina em cultivo em meio líquido}

Para estabelecer a relação entre o crescimento da biomassa e a produção de $\mathrm{N}$ acetilglicosamina no cultivo em estado sólido foi necessário empregar o método indireto de realizar o cultivo em meio líquido, para o qual a presença dos sólidos diluídos não contribuiria para a biomassa fúngica em suspensão. Na Figura 3.1, são apresentados os resultados da concentração de micélio seco obtido em cultivo líquido em função do tempo, onde a tendência de aumento da concentração de biomassa ao longo do tempo de fermentação, assim como as fases de crescimento podem ser visualizadas.

Na Figura 3.2 é apresentada a curva de conversão do teor de glicosamina a biomassa para o fungo M. thermophila M.7.7 obtida pela diluição em várias concentrações do 
micélio seco do fungo obtido em cultivo líquido após $48 \mathrm{~h}$ de cultivo. O comportamento da curva é tipicamente linear e o coeficiente de determinação $\left(R^{2}\right)$ da regressão linear foi muito elevado, igual a 0,996 . O fator de conversão entre glicosamina e biomassa seca foi de $6,39 \mathrm{mg}$ de glicosamina/g de biomassa, supondo-se que a concentração de glicosamina não varia com a idade do micélio.

Figura 3.1. Cinética de crescimento do fungo Myceliophthora thermophila M.7.7 em cultivo líquido através da medida do peso seco.

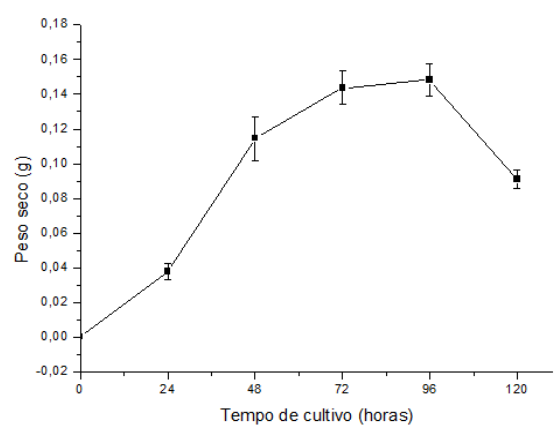

Figura 3.2. Curva de calibração entre a biomassa e o teor de glicosamina da biomassa de Myceliophthora thermophila M.77 obtido por cultivo submerso, após $48 \mathrm{~h}$ de cultivo.

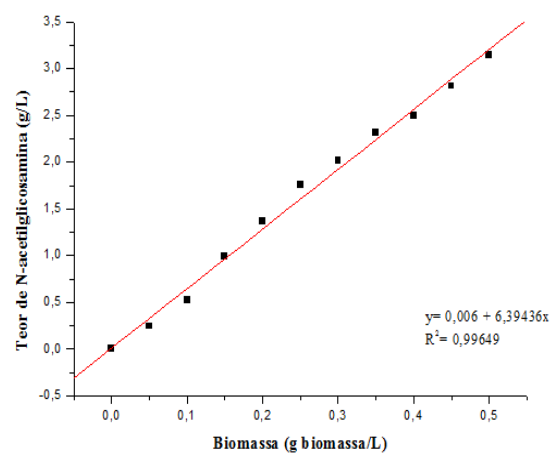

Essa mesma correlação entre o conteúdo de glicosamina e a biomassa seca obtida por cultivo líquido, foi estabelecida por outros autores, que fizeram uso dessa metodologia indireta para determinação da massa celular nos cultivos em meio sólido, como nos trabalhos de Aidoo et al. (1981), Bandelier et al. (1997), Gelmi et al. (2000), Scotti et al. (2001), Fenice et al. (2003) e Kalogeris et al. (2003).

\subsection{Análise de variabilidade na determinação do teor de $\mathrm{N}$ - acetilglicosamina em cultivo sólido}

Foram realizados testes para avaliar a variabilidade de ensaios repetidos de cultivo e de amostragem para a determinação de glicosamina. Para os primeiros, foram feitos três ensaios independentes em embalagens contendo $5 \mathrm{~g}$ de solido seco, sendo de cada um retiradas dez amostras de cerca de $0,5 \mathrm{~g}$ do material fermentado para as análises de glicosamina, para os tempos de 72 e $96 \mathrm{~h}$ de fermentação. Aos dados foi aplicado o modelo linear generalizado, sendo comparação entre médias feita através do teste de Tukey e a comparação entre variâncias pelo teste de Levene. Após a aplicação do modelo, foram constatadas duas amostras com resíduos elevados, que foram removidas do conjunto para ambos os tempos. A Tabela 3.2 - Análise estatística para os ensaios de repetição $\left(^{*}\right)$ - resume estas análises, onde se observa que não há diferença significativa entre as razões de massa de biomassa/massa de fermentado $(\mathrm{X})$ e há homocedasticidade entre os tratamentos. A menos das amostras do ensaio 3 para 96h, os coeficientes de variação (CV) foram abaixo de $16 \%$, apontando baixa variabilidade deste tipo de experimento. 
Tabela 3.2. Análises estatísticas para os ensaios de repetição $(*)$.

\begin{tabular}{ccccccc}
\hline \multicolumn{6}{c}{ X (mg biomassa/ g meio fermentado) } \\
\hline Ensaio & Média & $\mathbf{7 2 h}$ & $\begin{array}{c}\text { CV } \\
(\%)\end{array}$ & Média & IC & $\begin{array}{c}\mathbf{C V} \\
(\%)\end{array}$ \\
\hline 1 & $2,025^{\mathrm{aA}}$ & 0,278 & 16,4 & $1,744^{\mathrm{aA}}$ & 0,139 & 9,6 \\
2 & $1,852^{\mathrm{aA}}$ & 0,180 & 11,6 & $1,764^{\mathrm{aA}}$ & 0,178 & 12,2 \\
3 & $1,657^{\mathrm{aA}}$ & 0,217 & 12,5 & $1,749^{\mathrm{aA}}$ & 0,410 & 22,3 \\
\hline
\end{tabular}

Levene statistics $=0.659 ;$-value $=0.235$

(*) Letras minúsculas referem-se à comparação entre cultivos; letras maiúsculas referem-se à comparação entre tempos de cultivo. Letras iguais não diferem significativamente. IC - intervalo de confiança; CV - coeficiente de variação. Fonte: Autor (2015).

Tais testes são necessários devido ao caráter heterogêneo do substrato, composto por bagaço de cana de acúcar e farelo de trigo. $\mathrm{O}$ bagaço tem duas frações com composições químicas muito distintas, a casca e a medula, que diferem significativamente do farelo de trigo em composição, forma e dimensão. Deve-se destacar que não houve nenhum tratamento prévio do farelo de trigo e que o bagaço de cana foi apenas peneirado. Acredita-se que o fungo colonize preferencialmente o farelo de trigo como fonte primária de nutrientes, o que foi comprovado através da análise da cinética de secreção de enzimas.

\subsection{Cinética de crescimento fúngico através da técnica de determinação de $\mathrm{N}$ - acetilglicosamina}

O modelo logístico foi ajustado à média dos dados experimentais para cada tempo de cultivo, resultando em um coeficiente de determinação razoável $\left(\mathrm{R}^{2}=0,84\right)$, conforme apresentado na Figura 3.3a. Note-se que o ajuste não representa adequadamente a fase final do processo e subestima claramente o máximo de biomassa no sistema. Isto ocorre porque o modelo logístico não é adequado à representação das fases lag e de declínio da biomassa. Este modelo assume que, após atingir o máximo de densidade populacional, há um equilíbrio entre as taxas de natalidade $\mathrm{e}$ de mortalidade de células, de modo que a taxa de crescimento torna-se nula. Na Figura 3.3a, o ajuste do modelo é nitidamente distorcido pelos dados obtidos após a biomassa ter atingido seu valor máximo, observado às $72 \mathrm{~h}$ de cultivo.

Para contornar esta deficiência, um novo ajuste foi realizado assumindo-se que após as $72 \mathrm{~h}$ a biomassa permaneceria constante, conforme é apresentado na Figura $3.3 \mathrm{~b}$, resultando em um coeficiente $\mathrm{R}^{2}=0,98$ sob a denominação Dados modificados. Notese que a maior alteração no valor nos parâmetros estimados foi no máximo de biomassa no sistema, que passou de 1,821 para 1,889 $\mathrm{mg}$ de glicosamina/g de fermentado, sendo os demais parâmetros praticamente inalterados. Assim, as previsões dos modelos de crescimento devem representar com maior fidelidade esta fase, o que é obtido com maior êxito na segunda alternativa de cálculo.

A Tabela 3.3 apresenta os valores dos parâmetros de ajuste obtidos, onde é possível observar que embora o ajuste com Dados modificados tenha tido maior coeficiente de correlação, o ajuste aos dados experimentais originais forneceu parâmetros cinéticos de crescimento foram praticamente as mesmas que quando empregados os parâmetros obtidos pelo segundo ajuste, além de que os erros dos parâmetros também não alteraram significativamente as previsões. Portanto, para os engenheiros de processos interessados em operar biorreatores de CES, as margens de erro dos parâmetros obtidos por quaisquer dos dois ajustes são irrelevantes 
Figura 3.3. Cinética de crescimento de biomassa de Myceliophthora thermophila M.7.7 estimada por quantificação de glicosamina, com ajustes pelo modelo logístico (a- Ajuste com dados originais; b - Ajuste com dados modificados).

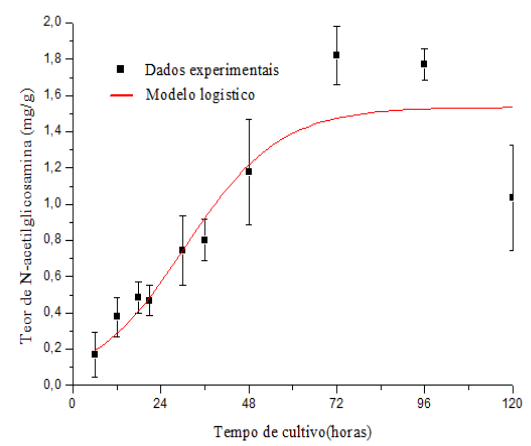

(a)

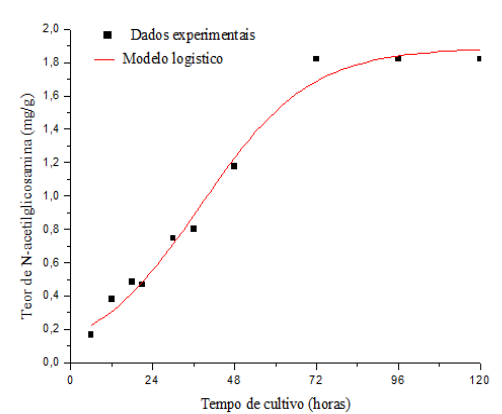

(b)

Tabela 3.3. Parâmetros cinéticos de crescimento obtidos para Myceliophthora thermophila M.7.7 (bagaço de cana e farelo de trigo 7:3 p/p, $70 \%$ de umidade, cultivo a $45^{\circ} \mathrm{C}$ ).

\begin{tabular}{|c|c|c|}
\hline Fungo & $\begin{array}{c}\text { Parâmetros } \\
\text { cinéticos }\end{array}$ & Valores \\
\hline \multirow{3}{*}{$\begin{array}{l}\text { Myceliophthora } \\
\text { thermophila M.7.7 } \\
\text { (Dados originais) }\end{array}$} & $X_{\text {máx }}$ & $\begin{array}{l}1,821, \mathrm{mg} \\
\text { biomassa } / \mathrm{g} \\
\text { fermentado }\end{array}$ \\
\hline & $\mathrm{X}_{0}$ & $\begin{array}{l}0,166 \quad \mathrm{mg} \\
\text { biomassa } \\
\text { fermentado }\end{array}$ \\
\hline & $\mu$ & $0,078 \mathrm{~h}^{-1}$ \\
\hline \multirow{3}{*}{$\begin{array}{l}\text { Myceliophthora } \\
\text { thermophila M.7.7 } \\
\text { (Dados modificados) }\end{array}$} & $X_{\text {máx }}$ & $\begin{array}{lr}1,88 \mathrm{mg} \\
\begin{array}{l}\text { biomassa } \\
\text { fermentado }\end{array} \\
\end{array}$ \\
\hline & $\mathrm{X}_{0}$ & $\begin{array}{l}0,160 \quad \mathrm{mg} \\
\text { biomassa } / \mathrm{g} \\
\text { fermentado }\end{array}$ \\
\hline & $\mu$ & $0,063 \mathrm{~h}^{-1}$ \\
\hline
\end{tabular}

Casciatori (2012) realizou ensaios com o fungo Trichoderma reesei $e$ utilizou o método de determinação de proteínas totais para estimativa indireta da biomassa, utilizando bagaço de cana e farelo de trigo 9:1 $\mathrm{m} / \mathrm{m}$ como substrato à $28^{\circ} \mathrm{C}$, e obteve um Xmáx de $21,7 \mathrm{mg} / \mathrm{g}$ e uma velocidade específica de crescimento de $0,05 \mathrm{~h}^{-1}$. Para a mesma linhagem, Trichoderma reesei cultivada em farelo de trigo à $28^{\circ} \mathrm{C}$, Smits et al. (1996) obtiveram velocidade específica de crescimento igual a $0,15 \mathrm{~h}^{-1}$ e Xmáx igual a $8,1 \mathrm{mg} / \mathrm{g}$.

A concentração da fonte de carbono e nitrogênio possui efeito significativo sobre a concentração máxima de biomassa e velocidade específica de crescimento. Presume-se que as diferenças encontradas neste trabalho comparadas com a literatura possa ser atribuída à diferença na composição dos substratos, tendo em vista que o bagaço de cana é um substrato composto por celulose, hemicelulose e lignina, o que limita a velocidade de crescimento do fungo quando comparado ao farelo de trigo puro e a glicose que são fontes mais facilmente assimiláveis.

\subsection{Análise da atividade metabólica através da atividade enzimática}

A Figura 3.4 representa a dinâmica da biossíntese das enzimas e demonstra que a atividade microbiana pode ser inferida pela análise dos metabólitos primários secretados, mas que há grande dificuldade em correlaciona-la diretamente com o crescimento fúngico, uma vez que não há um metabólito específico que permaneça no meio reacional sem ser degradado ao longo do processo e cuja concentração seja diretamente proporcional ao aumento da biomassa. Notese que a atividade da protease atinge seu máximo em $48 \mathrm{~h}$ de ensaio, seguido pela $\alpha$ amilase, em $72 \mathrm{~h}$, e pelas endoglucanases e xilanase às $96 \mathrm{~h}$. 
Figura 3.4. Atividade enzimática de proteases, amilases, xilanases e CMCases produzida pelo fungo Myceliphthora thermophila M.7.7 em cultivo no estado sólido ao longo de 120 horas.

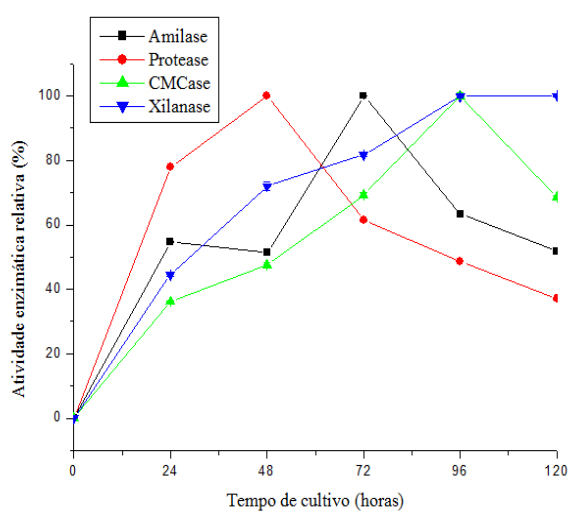

A heterogeneidade do meio de cultivo, possivelmente estimulou o microrganismo a usar um pool de fontes de nitrogênio, carbono, fósforo e micronutrientes do meio sólido, principalmente do farelo de trigo, para a produção de enzimas extracelulares. Segundo a literatura, o farelo de trigo contém um elevado teor de proteína, amido, açúcares e fibras, enquanto que o bagaço de cana é composto basicamente por celulose, hemicelulose e lignina. Desta forma, as $\alpha$ amilases e as proteases são esperadas ser secretadas com maior intensidade nos estágios iniciais do processo fermentativo, por serem fontes de carbono e nitrogênio facilmente assimiláveis. Quanto às proteases, há ainda a possibilidade de enzimas secretadas pelo próprio microrganismo. Com o decréscimo da quantidade de fontes de carbono facilmente assimiláveis, intensifica-se a secreção de celulases e hemicelulases, dentre elas a endoglucanase e a xilanase, que responderiam pela degradação da celulose e da hemicelulose presentes no farelo de trigo e no bagaço de cana.

À exceção da xilanase, as demais enzimas têm queda de atividade após atingirem o máximo, indicando possível ação das proteases ou outros inibidores. Couri et al. (1993) observaram comportamento similar para a produção de poligalacturonase em cultivo de Aspergillus niger 3T5B8 em farelo de trigo. Os autores relacionaram o comportamento da enzima com a possibilidade de produção de proteases após o seu ponto máximo, provocando a hidrólise e consequente diminuição de sua atividade.

\section{CONCLUSÕES}

A quantificação da biomassa através do teor de $\mathrm{N}$-acetilglicosamina demonstrou ser um bom indicativo do crescimento de Myceliophthora thermophila M.7.7 em cultivo sólido. A atividade enzimática representou a dinâmica da biossíntese das enzimas e demonstrou que a atividade microbiana pode ser inferida pela análise dos metabólitos primários secretados, mas não foi possível correlaciona-la diretamente com o crescimento fúngico, devido ao acompanhamento de proteases.

\section{NOMENCLATURA}

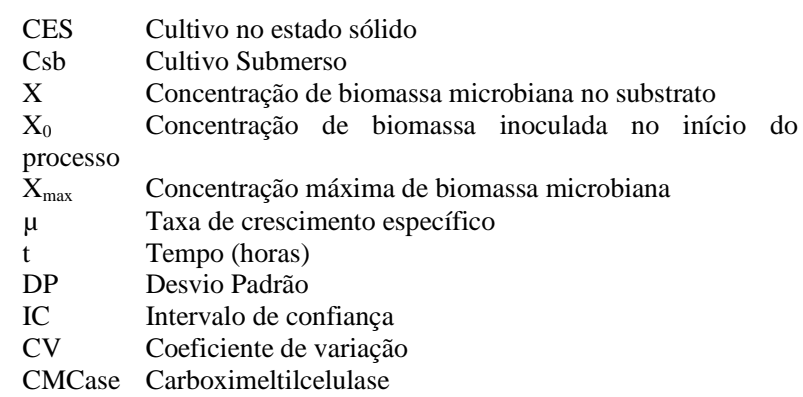

\section{REFERÊNCIAS}

AIDOO, K. E., HENDRY, R., WOOD, B. J. Estimation of fungal growth in a solid state fermentation system. Applied Microbiology and Biotechnology, v. 12, p. 6-9, 1981.

BANDELIER, S.; RENAUD, R. ; DURAND, A. Production of gibberellic acid by fed-batch solid state fermentation in an aseptic pilot- 
scale reactor. Process Biochemistry, v. 32(2), p.141 - 145, 1997.

CASCIATORI, F. P. Biorreatores de leito fixo para fermentação em estado sólido: ampliação de escala para produção de celulases de origem fúngica. 2012. Tese (Doutorado), Universidade Estadual Paulista, São José do Rio Preto, Brasil.

COURI, S. Efeito de cátions na morfologia do agregado e na produção de poligalacturonase por Aspergillus niger mutante 3T5B8. 1993. Tese (Doutorado). Universidade Federal do Rio de Janeiro, Rio de Janeiro, Brasil.

DALSENTER, F. D. H. Efeito da temperatura na cinética de crescimento de Rhizopus oryzae em cultivo no estado sólido. 2005. 136 p. Tese (Doutorado). Universidade Federal do Paraná, Curitiba, 2005.

FANAEI, M. A.; VAZIRI, B. M. Modeling of temperature gradients in packed-bed solidstate bioreactors. Chemical Engineering and Processing, v. 48, p. 446-451, 2009.

FENICE, M.; SERMANNI, G.G. ; FEDERICI, F. ; D'ANNIBALE, A. Submerged and solid- state production of laccase and Mn-peroxidase by Panus tigrinus on olive mill wastewater- based media. Journal of Biotechnology, v.100, p.77 - 85, 2003.

GELMI, C.; PÉREZ-CORREA, R. ; GONZÁlEZ, M. ; AGOSIN, E. Solid substrate cultivation of Gibberella fujikuroi on inert support. Process Biochemistry, v.35, p.1227- 1233, 2000.

GHOSE, T.K. Measurement of cellulase activities. Pure \& App. Chem., v. 59, p. 257-268, 1987.
KALOGERIS, E. ; INIOTAKI, F. ; TOPAKAS, E. ; CHRISTAKOPOULOS, P. ; KEKOS, D. ; MACRIS, B.J. Performance of an intermittent agitation rotating drum type bioreactor for solid-state fermentation of wheat straw. Bioresource Technology, v. 86, p.207-213, 2003.

LONSANE, B.K. ; RAIMBAULT, M. ; VINIEGRA-GONZALEZ, G. (Eds.) Advances in Solid State Fermentation. Dordrecht: Kluver Academic Publishers, p. 93-111, 1999.

MERHEB, C.W; CABRAL, H; GOMES, E; DA SILVA, R. Partial characterization of protease from a thermophilic fungus, Thermoascus aurantiacus, and its hydrolytic activity on bovine casein. Food Chemistry. v.104, p. 127-131, 2007.

SCOTTI, C.T. ; VERGOIGNAN, C. ; FERON, G. ; DURAND, A Glucosamine measurement as indirect method for biomass estimation of Cunninghamella elegans grown in solid state cultivation conditions. Biochemical Engineering Journal, v.7, p.1 5, 2001.

SMITS, J.P.; RINZEMA, A. ; TRAMPER, J.; VAN SONSBEEK, H.M. ; KNOL, W. Solidstate fermentation of wheat bran by Trichoderma reesei QM9414: substrate composition changes, $\mathrm{C}$ balance, enzyme production, growth and kinetics. Applied Microbiology Biotechnology, v. 46, p.489498, 1996.

\section{AGRADECIMENTOS}

À CAPES pelo apoio financeiro. 